\title{
El terrorismo y los medios de comunicación: un enfoque traductológico (EN-ES)
}

\author{
Alba Montes Sánchez \\ Universidad de Córdoba \\ 132mosaa@uco.es \\ https://dx.doi.org.10.12795/futhark.2019.il4.06
}

Fecha de recepción: 12.09 .2018

Fecha de aceptación: 25.01.2019

Resumen: El terrorismo, un problema de repercusión mundial, se ha convertido en una forma de violencia verdaderamente compleja de delimitar. El presente estudio aborda el léxico y la terminología del ámbito del terrorismo desde una perspectiva traductológica, con el objetivo fundamental de analizarlos terminológicamente en inglés y aplicar su traducción al español. Para ello, partimos de un breve análisis sobre el tratamiento que hacen los medios de comunicación de este léxico, así como de las expresiones que derivan de este tipo de discursos. Consideramos que la homogeneización de esta terminología y su correcto equivalente en español, podrían ser de utilidad para profesionales que trabajan en la investigación o en la transferencia de información sobre contenidos terroristas.

Palabras clave: medios de comunicación, terminología, terrorismo, traducción, transferencia de la información.

\section{Terrorism and the media: a translational approach (EN-ES)}

Abstract: Terrorism, a problem of global repercussion, has become a truly complex form of violence to define. The present study presents the lexicon and terminology of the field of terrorism from a translation perspective, with the fundamental objective of analyzing them terminologically in English and applying their translation into Spanish. In order to develop this, our basis is a brief analysis of the media's treatment of this lexicon, as well as the expressions that derive from this type of discourses. We consider that the homogenization of this terminology and its correct equivalent in Spanish could be useful for professionals working in the research or in the transfer of information on terrorist content.

Keywords: media, terminology, terrorism, translation, transfer of information. 
Sumario: Estado de la cuestión y planteamiento del estudio. I. El terrorismo y los medios de comunicación. 2. La traducción y el terrorismo internacional. El rol del traductor. 3. El corpus. 4. El término attack. Análisis y propuestas de traducción. Conclusiones

\section{Estado de la cuestión y planteamiento del estudio}

En la Europa actual el derecho de las personas a circular y residir libremente dentro de la Unión Europea se considera la piedra angular de la ciudadanía con el objeto de reforzar así los vínculos sociales, políticos, económicos y culturales entre países. No obstante, en los últimos años se han sucedido en el continente europeo ataques en contra de la libertad, derecho fundamental del ciudadano, perpetrados por organizaciones y grupos terroristas que continúan siendo una amenaza en la actualidad. Por ello consideramos que la traducción y la interpretación ocupa un lugar crucial en la prevención y lucha contra el terrorismo por tener como finalidad la transmisión de datos y de información de forma objetiva y neutral.

El terrorismo, un problema de repercusión mundial, se ha abordado desde numerosos ámbitos de estudio, destacando trabajos sobre el concepto de terrorismo global y yihadismo (Cañete 2017; Jordán 2009; Kaldor 2003; Lobato de Enciso 2017; López Calera 2002; Milosevich 2006; Powell 2009, Reinares 2004 y 2008; Revilla 2005; Rodríguez et. al. 2017; Torres y Jordán 2013; Savater 2004). Sin embargo, los trabajos relacionados con la terminología, la traducción o la lingüística aplicada son escasos en esta área.

Hoy, los gobiernos y organizaciones internacionales contemplan el terrorismo como un factor global, complejo $y$, en cierto modo, sorpresivo, por lo que, consecuentemente, entre sus medidas para prevenir nuevos atentados terroristas se encuentra la figura del traductor-intérprete como pieza fundamental para acceder a la información.

Asimismo, el léxico empleado por los medios de comunicación al abordar contenido relacionado con el terrorismo ha suscitado un gran interés en las investigaciones que hemos desarrollado recientemente. Por ello, para la realización de nuestro estudio partimos de la siguiente hipótesis: el conocimiento terminológico y la competencia traductora influyen de forma directa en la transferencia de la información a través de los medios de comunicación.

La finalidad del presente estudio es, por una parte, presentar la relación existente entre los medios de comunicación y el fenómeno terrorista a través de cuestiones lingüísticas y destacar el rol del traductor en estos contextos; por otra, contrastar el tratamiento de la terminología terrorista entre documentos de carácter institucional y fuentes periodísticas, con el objeto de estudiar si existe una homogeneización de este léxico y evidenciar que el establecimiento de 
equivalencias en la lengua meta conlleva enfrentarse a determinados dilemas traductológicos.

\section{El terrorismo y los medios de comunicación}

Con el fin de establecer un vínculo entre la acción terrorista y los principales canales de difusión de la información, partimos de la publicación de Veres (2006), El signo perverso: sobre lenguaje, terrorismo y práctica periodística, para destacar como aspecto primordial la adherencia y atracción que los terroristas sienten hacia los medios de comunicación.

Autores como Eco (1978) señalan que "el terrorismo es un fenómeno de nuestra época, de la época de los medios de comunicación de masas", hasta el punto de afirmar que "si no hubiera medios masivos, no se producirían estos aspectos destinados a ser noticias". El hecho de asegurar que sin los medios de comunicación o mass media no existiría el terrorismo, o, como dice Rodrigo (1991: 27), que "el terrorismo es rehén de los media y estos lo son de él" hace cuestionar la delimitación del concepto "terrorismo" y de los diferentes tipos de terrorismo que existen. En cierto modo, el tipo de terrorismo - en concreto el yihadismo que nos azota en la actualidad logra sus objetivos gracias, en gran parte, a la información facilitada a través de los medios de comunicación, pues otorgan cobertura inmediata sobre sus actuaciones a nivel planetario, lo que da a entender, en palabras de Veres (2006: 290) que "resulta bastante cierta esa relación entre medios y terrorismo, ya que mientras esa información no resulta publicada, los atentados terroristas no son noticia".

Los medios de comunicación y su manera de informar sobre el terrorismo han contribuido de forma muy significativa a paralizar y ofuscar todavía más la eterna política de definir al autor de los actos de violencia como terrorista 0 como combatiente por la libertad (Hoffmann, 1990: 4I).

Aquí hacemos hincapié en que el fenómeno terrorista presenta numerosos problemas de tipo lingüístico en relación con los medios de comunicación. El lenguaje, así como la terminología que se emplea en este tipo de discursos, debe ser tratada con especial tiento y cuidado, puesto que, como menciona Veres (2006: 292), la emisión de una noticia en cualquier soporte supone un acto semejante a cualquier otra acción humana, pero en el caso de tratarse de una noticia sobre contenido terrorista, el riesgo aumenta respecto a otras noticias informativas. Debemos tener en cuenta que, en esta materia en concreto, reproducir de forma exacta los vocablos empleados por los terroristas sería sinónimo de legitimar sus actos; es decir, en múltiples casos estos grupos y organizaciones terroristas se sirven de un léxico con connotaciones bélicas para justificar sus acciones, por lo que al transmitir esta información empleando esa terminología se denota parcialidad, en lugar de objetividad. Por ello, tanto el tratamiento de esta 
terminología como su traducción son cuestiones que no deben atender solo a la objetividad, sino también al emisor y al contexto político, social y cultural en el que se enmarcan.

\title{
2. La traducción y el terrorismo internacional. El rol del traductor
}

Los atentados terroristas del II de septiembre de 20 I I en Nueva York, así como los del II de marzo de 2004 en Madrid, obligaron a los estados democráticos occidentales a adoptar medidas legislativas contra el fenómeno terrorista para endurecer las penas aplicadas a este tipo de delitos. Estas medidas han sido reforzadas tras los ataques perpetrados en París el 13 de noviembre de 2015 y el atentado al Mercado de Navidad de Berlín el 19 de diciembre de 2016, entre otros. Igualmente, estos ataques evidenciaron la relevancia de la traducción en las investigaciones llevadas a cabo por los servicios de inteligencia y la importancia del intérprete en juicios, interrogatorios e investigaciones.

\begin{abstract}
Podemos sugerir que la ingente cobertura periodística de los grandes atentados del II-S y II-M ha servido, de forma indirecta, para llamar la atención sobre el papel destacado que cumplen los traductores y los intérpretes en la resolución de conflictos y también de su capacidad para evitarlos. [...] Es importante destacar en este contexto el interés que en los últimos años ha despertado en los Estudios de Traducción la figura del traductor como agente de intervención social en contextos geopolíticos. Conforme la globalización y los conflictos bélicos superan las fronteras lingüísticas, culturales y nacionales, resulta sintomático el desarrollo cada vez más frecuente de estudios sobre sociología de la traducción y la atención a los aspectos éticos de su actividad (Pegenaute 2012: 405).
\end{abstract}

Pegenaute ha sido uno de los pocos autores que ha centrado su investigación en la relación o vinculo existente entre los profesionales de la traducción y la interpretación y el terrorismo. El autor sitúa como punto de partida la globalización (2012: 398), la cual ha aportado numerosos avances positivos con respecto cuestiones sociales, políticas, tecnológicas y económicas. No obstante, desde una perspectiva muy poco optimista, este autor expone que la globalización ha traído "consecuencias colaterales negativas", ya que ha favorecido la perdida de la identidad cultural local (o la creación de una identidad más internacional) y el desarrollo del terrorismo internacional, definido por Calduch Cervera (1993: 343) como "aquella forma de terrorismo que de un modo directo se desarrolla en o afecta a diversos países, sus ciudadanos y/o territorios, o a los representantes de organizaciones intergubernamentales”.

Según Brooks (2016), en la actualidad, el gobierno estadounidense es el mayor empleador de traductores, contratando lingüistas expertos que también se ocupan de la lectura de los textos meta, para así analizar todos los matices del texto origen. De acuerdo con Pegenaute (2012: 399-40I), el gobierno 
estadounidense priorizó tras los atentados del II-S el ámbito lingüístico y cultural como pieza clave para salvaguardar la seguridad nacional, así como para consolidar su lugar como potencia económica en el panorama internacional. De hecho, en España no se había priorizado el ámbito lingüístico antes de los atentados de 2004, lo que se evidenció en problemas de contrainteligencia desarrollados antes, durante y después del II-M, siendo material muy relevante para la investigación: conversaciones telefónicas no traducidas que permanecieron durante meses en cintas grabadas; comunicaciones de presos islamitas, destrucción de cintas con contenido en un dialecto específico del árabe o ausencia de intérpretes en las instituciones penitenciarias, entre otros (2012: 402-404).

La labor que desempeña el traductor en cuestiones relacionadas con el terrorismo resulta semejante a lo expuesto por Veres (2006). Según este autor, la profesión del periodista, en este contexto quizás más que en otros, conlleva una gran responsabilidad y compromiso social, ya que el traslado de la información proveniente de grupos y organizaciones terroristas y transmitirla a la ciudadanía de forma completamente objetiva podría desencadenar graves consecuencias, como mínimo sociales. Lo mismo ocurre con la tarea del traductor en este contexto, pues esta situación genera una serie de interrogantes éticos sobre el rol que debe desempeñar tanto el lingüista como el informador con su principal herramienta de trabajo: el lenguaje. Como señala Casado (1991: 56), “las palabras no determinan nuestra forma de pensar, pero de alguna forma nos predisponen a favor de ciertas líneas de pensamiento".

De acuerdo con Shariffan (2007), es preciso destacar la responsabilidad ideológica y sociopolítica que supone el tratamiento de determinadas palabras en contextos en los que la carga ideológica está muy presente. Shariffan expone ejemplos de palabras como "compromiso", "concesión", "yihad" o metáforas como "Eje del mal”, que, al ser tratadas de forma errónea en el proceso de traducción o interpretación, podrían ocasionar graves consecuencias políticas o sociales, pues están cargadas de elementos culturales.

Por tanto, recurrimos a la afirmación de Robles (2007: 33), quien asegura que los traductores se responsabilizan de actuar como "fuerza de choque" de las investigaciones en esta materia, además de constituir un elemento indispensable y crucial en determinadas situaciones en las que se requieren sus servicios con inminente urgencia: interpretaciones telefónicas, traducción de material propagandístico destinado a la radicalización o actuar como agente de intervención social. En este sentido, podríamos afirmar que la figura del traductor-interprete resulta crucial para lograr que el análisis, la consulta, la transmisión y la verificación de la información sea correcta y veraz. El autor coincide con Brooks (2016), quien sostiene que el rol de estos profesionales se ha ido moldeando con el paso de los años hasta la actualidad, en la que los consideramos de necesaria relevancia para 
hacer frente al terrorismo y poder mantener las relaciones políticas de carácter internacional: "hoy, necesitamos la práctica traductológica más que nunca".

\section{El corpus}

En primer lugar, creemos oportuno determinar qué es y qué función tiene un corpus monolingüe. Según Kenny, (200I: 58), un corpus monolingüe es aquel que está integrado por una sola lengua y que a su vez puede subdividirse entre los corpus monolingües traductológicos u originales (Laviosa, 2002: 35). El corpus que compilamos se trata de un corpus monolingüe original o no traducido, ya que está compuesto por textos especializados que se han usado sobre todo para la extracción de terminología.

Los textos compilados se corresponden con el EU Terrorism Situation \& Trend Report ${ }^{1}$ (en adelante, TE-SAT), traducido al español como "Informe de la situación y tendencias del terrorismo", elaborado y publicado anualmente Europol, agencia de la Unión Europea. Este informe, publicado exclusivamente en inglés, proporciona una visión general del fenómeno terrorista en la UE desde el año 2007 hasta la actualidad. Como ya hemos mencionado con anterioridad, la lucha contra el terrorismo encabeza la lista de prioridades de los líderes e instituciones europeas, por lo que Europol presenta el TE-SAT como uno de los análisis estratégicos que más contribuye a la defensa y protección de la región europea.

Este informe ofrece datos y cifras específicas sobre el terrorismo en la UE a los funcionarios del orden público, responsables políticos y a la ciudadanía en general, a la vez que identifica tendencias en desarrollo en este ámbito y presenta los principales promotores, impulsores o "imanes" de la comisión de actos terroristas. El TE-SAT es elaborado por expertos de Europol, quienes se apoyan y complementan con información y datos cuantitativos y cualitativos proporcionados por los Estados miembros de la UE y Eurojust. Desde su primer lanzamiento en 2007, la estructura del TE-SAT ha seguido un patrón muy similar. Mencionamos como ejemplo el informe publicado en 2018, el cual estaba integrado por los siguientes apartados: prólogo, tendencias, visión general, terrorismo yihadista, terrorismo etno-nacionalista y separatista, terrorismo de izquierdas y anarquista, terrorismo de derecha y terrorismo single-issued (individual). Además, el TE-SAT ofrece como conclusión un conjunto de siete anexos entre los cuales se insertan estadísticas sobre los ataques fallidos, frustrados y completados en el año 2019 a escala nacional y clasificados según el tipo de terrorismo, estadísticas de las detenciones nacionales según el tipo de terrorismo, estadísticas sobre sentencias y penas por delitos de terrorismo, una visión general de las actuaciones antiterroristas desarrolladas por Europol, la metodología seguida para elaborar el

I En línea: https://www.europol.europa.eu/tesat-report [Fecha de consulta: 05/I2/20|8]. 
informe, las siglas y abreviaturas utilizadas, $y$ enmiendas legislativas en materia terrorista a escala nacional.

En suma, todos los textos siguen una símil estructura, constan de apartados iguales o muy parecidos y su extensión es muy regular, notándose así que esta ha aumentado considerablemente en los últimos años.

\section{El término attack. Análisis y propuesta de traducción}

El empleo de anglicismos es cada vez mayor en castellano, por lo que, en el ámbito que concierne al terrorismo y a otros tipos de violencia de carácter internacional, esta práctica también está muy presente. Como resultado, la parte práctica del presente artículo gira en torno a la traducción del término attack y de sus diferentes construcciones léxicas.

Durante el proceso de traducción del término attack al español, debemos establecer la diferenciación entre "ataque" y "atentado". "Ataque" es un término genérico que aparece frecuentemente en textos sobre esta materia debido, quizás, al calco que realizamos en el proceso de traducción del inglés al español del término attack. La Fundéu recuerda que en español ya disponemos de una palabra para referirnos a este tipo de actos, por ello, aunque el empleo de la expresión "ataque" o "ataque terrorista" no es incorrecto, es preferible recurrir a la construcción "atentado terrorista" en la lengua meta. De igual forma ocurre con ejemplos como "ofensiva terrorista", posiblemente por tratarse también del gran influjo que ejerce la lengua inglesa (terrorist offense).

El término attack aparece un total de 667 veces en los textos compilados en nuestro corpus y en diferentes tipos de construcciones léxicas, destacando así las siguientes:

- $[\ldots]$ and terrorist organisation and on the charge of preparing for an armed attack and recruiting for the jihad. Not all members of the The UK [...] (TE-SAT 2007).

- [...] terrorist group called Rivolta Anonima Tremenda claimed responsibility for a double attack against a police station in the town of Fossano in Italy. The [...] (TE-SAT 2007).

- $[\ldots]$ as roads and railways, and attacks targeting the police, including an IED attack against an off duty police officer, which resulted in serious injury.69 It can [...] (TE-SAT 2009).

- $[\ldots]$ to most Member States, despite the fact that only one Islamist terrorist attack - a bomb attack in Italy - took place in the EU in 2009. Islamist [...] (TE-SAT 20I0). 
- $[\ldots]$ debt burden and stem the impact of the economic crisis. The arson attack against a job centre in Austria can also be placed in this [...] (TE-SAT 20II).

- [...] airport by a religiously-inspired individual in March 201I is not a terrorist attack according to German legislation, although the incident [...] (TE-SAT 2012).

- In October 2012, a home-grown terrorist group responsible for a grenade attack against a kosher grocery in Paris (France) was dismantled [...] (TE-SAT 20I3).

- [...] to al-Qaeda were arrested in May 2013 for planning a car bomb attack against the French or the American ambassador in Cairo. They were released [...] (TE-SAT 2014).

- $\quad[.$.$] had links to AQAP in Yemen. The perpetrator of the Thalys$ train attack and a number of the suspects involved in the November Paris attacks [...] (TE-SAT 2016).

- [...] Deep Web is considerably larger than the Surface Web. A zero-day attack happens once a software/hardware vulnerability is exploited and attackers release [...] (TE-SAT 2016).

- $[\ldots]$ relatively small scale compared to the Nice attack. A similar soft targeted attack as in Nice took place on 19 December in Berlin, Germany. A truck [...] (TE-SAT 2017).

- $\quad[. .$.$] in 2016, UN troops and Mauritanian soldiers were killed.$ These included a joint attack by AQIM, Ansar al-Din and another local group on 21 July on [...] (TE-SAT 2017).

Con el objeto de analizar este término y sus distintas construcciones en mayor profundidad desde una perspectiva traductológica, creemos como un paso crucial tener en consideración parámetros como el término y su contexto. A nuestro parecer, el contexto es un apartado elemental para conocer cuándo y dónde se emplea y, por tanto, su correcta forma de aplicación en la LM a través del equivalente preciso. Por ello, partimos de un breve comentario en el que, a modo introductorio, analizamos la traducción del término propuesto, las dificultades que nos hemos encontrado en su traducción, la relevancia de determinados elementos culturales para su correcto significado o el uso los errores más comunes en su tratamiento, entre otros. Asimismo, hemos creído conveniente para el análisis recurrir a fuentes periodísticas con el fin de observar cómo se emplea el léxico analizado. Nuestro objetivo es facilitar la traducción y el tratamiento de esta terminología, por lo que decidimos apoyarnos en este tipo de información para 
demostrar que no existe una homogeneización del léxico en el ámbito periodístico, en concreto.

Posteriormente, exponemos una tabla con ejemplos dividida en dos claras partes: en el que la primera se analizan las versiones en inglés y en ella se incluye el término o la construcción en lengua inglesa [EN] junto con los contextos [1] y [2] extraídos de documentos pertenecientes al ámbito de la UE y de fuentes periodísticas, respectivamente. En la segunda parte, se presenta una propuesta de traducción al español de los contextos [I] y [2] presentados en la primera parte [EN].

\section{—Suicide attack}

Los denominados "ataques suicidas" o "atentados suicidas" se remontan a los siglos X, XI e incluso XVIII, cuando en varios países asiáticos se realizaba esta práctica para plantar cara a la presencia occidental. En la actualidad, este tipo de atentados se perpetra con mayor asiduidad, pues fue el grupo libanés Hezbollah quien en 1983 los potenció. Ciertas investigaciones sostienen que esta práctica se vincula con el concepto de "autosacrificio", pues los perpetradores no se consideran "suicidas, sino como mártires", ya que "el autosacrificio es una vía para legitimar su causa, generando un sentimiento de inspiración y prometiendo la gloria individual ${ }^{2}$ ".

\section{EJEMPLO 1}

[EN] ENTRY: Suicide attack

Contexto 1: [...] Pakistan in 2008 took place in the North-West Frontier Province. The suicide attack against the Marriott hotel in Islamabad on 20 September 2008 could be seen as [...].

Fuente: TE-SAT 2019

Contexto 2: Double suicide bomber attacks leave 1 dead, several hurts in Tunisia's capital

Fuente: Global NEWS, 27 de junio de 2019

Propuesta trad. contexto 1: [...] tuvo lugar en 2008 en la Provincia Fronteriza Noroccidental de Pakistán. El atentado suicida contra el hotel Marriott en Islamabad el 20 de septiembre de 2008 podría verse como [...].

Propuesta trad. contexto 2: Un doble atentado suicida con bomba deja un muerto y varios heridos en la capital de Túnez.

2 En línea: https://www.lahaine.org/internacional/atentados_suic.htm [Fecha de consulta: 10/06/2019] 
En este ejemplo, decidimos contrastar los titulares de dos medios de comunicación digitales españoles sobre la misma noticia, publicados el mismo día. En las siguientes imágenes podemos observar que la prensa española recurre a ambas construcciones, tanto "ataque" como "atentado suicida", mientras que en inglés únicamente hemos encontrado el empleo de la combinación léxica suicide attack:

\section{Muere un policía en un doble atentado suicida en Túnez}

- Dos atacantes han hecho estallar sendos artefactos contra la Policia en la capital

27.06.2019 I actualización 22.03 horas Por RTVE es

RTVE.es, 27 de junio de 2019

INTERNACIONAL
Estado Islámico reivindica el doble ataque suicida
en Túnez, que ha dejado como mínimo un policía
muerto

El Periódico, 27 de junio de 2019

\section{-Knife attack}

En primer lugar, nos gustaría destacar la intervención del secretario del Centro de la UNED en Ceuta, Enrique Ávila Pérez, en la Conferencia "Procesos de radicalización yihadista: España como referencia” celebrada en Córdoba, el pasado 20 de marzo de 2019. Ávila señala que es una tarea realmente compleja poder detener un atentado terrorista en la actualidad, pues el terrorismo al que nos enfrentamos puede actuar de formas tremendamente inesperadas. Esto quiere decir que las armas y los medios que emplean estos terroristas son muy económicos, podría incluso afirmarse que están al alcance de cualquiera, ya que es relativamente asequible el acceso a la compra de cuchillos, navajas u otro tipo de 
arma y posteriormente perpetrar crímenes en nombre de una organización terrorista, lo cual dificulta aún más la posibilidad de frenar un atentado.

Para la traducción de este ejemplo, es fundamental la consulta de diversas fuentes, así como atender a su contexto, con el fin de atender al equivalente que más se adecua en la LM.

\section{EJEMPLO 2}

[EN] ENTRY: Knife attack

Contexto 1: [...] drove a van into pedestrians on London Bridge and launched a knife attack after exiting the vehicle. The attack was claimed by IS [...].

Fuente: TE-SAT 2019

Contexto 2: One person is dead and nine more have been wounded in a knife attack near Lyon in France, while an Afghan asylum seeker has been detained.

Fuente: 9 NEWS, 1 de septiembre de 2019

Propuesta trad. contexto 1: [...] conducía una camioneta hacia los peatones del London Bridge y perpetró un ataque con arma blanca al salir del vehículo. El El reivindicó el atentado [...].

Propuesta trad. contexto 2: Una persona fallecida y otras nueve resultaron heridas a consecuencia de un ataque con arma blanca cerca de Lyon (Francia). Un refugiado afgano ha sido detenido.

En este caso, también creemos conveniente contrastar los titulares de dos periódicos digitales españoles sobre la misma noticia, publicados el mismo día. Como hemos mencionado con anterioridad, la multitud de combinaciones léxicas que ofrece el término attack hace que en la traducción al castellano nos surja la incógnita sobre qué término se adapta mejor a cada contexto, si "atentado" o "ataque". En el presente ejemplo, la gran parte de fuentes consultadas emplea "ataque", por lo que nos remontamos a lo fijado por la Fundéu y aceptamos el empleo de este término genérico en este caso concreto. Asimismo, mientras que en inglés únicamente hemos encontrado el empleo de la combinación léxica knife attack, en las siguientes imágenes podemos observar que en español se emplea tanto "ataque con cuchillo" como "ataque con arma blanca". El Diccionario del español jurídico define "arma blanca" como "un arma constituida por una hoja metaliza y otro material de características físicas semejantes, cortante o punzante". Este término en inglés puede encontrarse como knife o como bladed weapon, por lo que en nuestra propuesta de traducción decidimos recurrir a la opción de "arma 
blanca" ya que no se especifica en el cuerpo de la noticia de qué tipo de arma en concreto se trata:

\section{Erconitaencal}

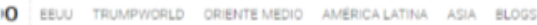

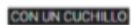

El Estado Islámico asume la autoría del ataque con cuchillo en Melbourne

El Confidencial, 9 de noviembre de 2018

ABL internacional

España Internacional Economiav Sociedad Madrid * Familiav Opinión * Deportesv Gentev Cultura * Ciencia Historia Viajar * Play* boliodad

Un muerto y dos heridos tras un ataque con arma blanca en Melbourne

ABC Internacional, 9 de noviembre de 2018

-CBRN attack

El Ministerio de Defensa describe la sigla CBRN como "armas químicas, biológicas, radiológicas y nucleares”. Según el LISA Institutite ${ }^{3}$, un atentado CBRN hace referencia a ataques terroristas en los que se utiliza material nuclear, biológico, químico o radiológico, comúnmente conocidas también como armas de destrucción masiva o bombas sucias, puesto que este tipo de armas o de ataques provocan un gran impacto, tanto en número de víctimas como en su capacidad de destrucción.

Tras haber consultado textos que emplean estas siglas, evidenciamos que se hace recurre generalmente a la sigla correspondiente a la descripción en inglés:

3 En línea: https://www.lisainstitute.com/blogs/blog/como-actuar-atentado-nbqr-nuclear-biologicoquimico-radiologico [Fecha de consulta: 06/07/2019]. 
- "[...] por miembros de al-Qaeda en Iraq, entre 2006 y 2007, no ha habido en el mundo ningún incidente grave de tipo químico, biológico, radiológico o nuclear (CBRN)". El Imparcial, 20 de marzo de 2012.

- "El Batallón de Defensa Química, Biológica, Radiológica y Nuclear (CBRN) de la OTAN representa tanto una solución eficaz ante un déficit de capacidades como un modelo para la transformación de la Alianza”. Revista de la OTAN, 2005.

- "Se trata de una iniciativa de la Comisión Europea para que los Veintisiete desarrollen nuevos sistemas de detección de amenazas químicas, biológicas, radiológicas o nucleares (QBRN) y refuercen sus sistemas de comunicación e intercambio de información". Elmundo.es, 15 de noviembre de 2010.

No obstante, en el último ejemplo observamos como no se recurre a la forma original en lengua inglesa, sino que se emplean las siglas correspondientes a la descripción del término en español. Proponemos, por tanto, emplear las iniciales correspondientes a las palabras que componen esta sigla en español. Como podemos apreciar, únicamente variaría la primera letra, lo que mantendría un patrón muy similar a la forma originaria en inglés, y aun así se adaptaría a la descripción de la sigla en español. De esta forma, aprovecharíamos que los tres términos restantes coinciden en la letra inicial y no emplearíamos nos serviríamos de una forma abreviada extranjera cada vez que hagamos uso de esta sigla en nuestra lengua.

\section{EJEMPLO 3}

[EN] ENTRY: CBRN attack

Contexto 1: The likelihood of a CBRN attack occurring is assessed as being low, but the consequences of such an incident remain serious.

Fuente: TE-SAT 2012

Contexto 2: "Extremists remain interested in CBRN materials. However, alternative methods of attack such as employing firearms or conventional explosive devices remain far more likely."

Fuente: https://www.independent.co.uk/news/uk/home-news/issis-terrorattacks-uk-plots-supporters-travel-syria-frustrated-basu-foreign-fightersa8576431.html

Propuesta trad. contexto 1: La probabilidad de que se perpetre un atentado QBRN es baja, pero las consecuencias de tal incidente continúan hoy siendo graves. 
Propuesta trad. contexto 2: "Los extremistas continúan interesados en los materiales QBRN. No obstante, prevalecen las formas de atentado alternativas como el uso de armas de fuego o de artefactos explosivos convencionales".

\section{Conclusiones}

Tras este breve estudio, podemos extraer una serie de conclusiones acerca de la relevancia del profesional en Traducción e Interpretación en materia terrorista, así como conocer más de cerca los conceptos que se asocian con este ámbito y su terminología.

En primer lugar, hemos confirmado la hipótesis inicial planteada. El conocimiento terminológico, las tareas de documentación y la resolución de determinados dilemas traductológicos son de vital importancia para trasladar la información de forma adecuada. Asimismo, se debe atender a la competencia lingüística de los profesionales que tratan con el léxico relacionado con el terrorismo, en particular, y con otras formas de violencia, en general, pues como hemos evidenciado a lo largo del presente estudio, en el fenómeno del terrorismo están implicados no solo factores sociales, jurídicos o políticos, sino también culturales.

No obstante, debemos destacar la carencia de recursos terminológicos monolingües, bilingües o multilingües en este ámbito de estudio. Consideramos que la búsqueda de información terminológica que nos ayudara a esclarecer los dilemas planteados durante el análisis es una de las mayores dificultades del estudio. Se echa en falta una mayor variedad de recursos en materia de terrorismo, así como disponer de una mayor accesibilidad a glosarios o diccionarios etimológicos sobre este ámbito, especialmente disponibles en español, con el mero fin de atender a cuestiones terminológicas y traductológicas

Finalmente, creemos que sería conveniente mencionar que el uso del léxico está directamente asociado a los conceptos y, por tanto, a los estereotipos que se crean en la sociedad, lo que supone un aliciente más para el correcto estudio y empleo de la terminología relacionada con el terrorismo, así como con cualquier tipo de violencia. Por ello, pretendemos que nuestro breve estudio enfocado en la traducción y la terminología pueda transmitir los matices sociales o culturales que caracterizan al léxico analizado, lo que podría redundar en beneficio para traductores e intérpretes y otros profesionales, como los periodistas encargados de transmitir información a través de los medios de comunicación. 


\section{Referencias bibliográficas}

Brooks, R. (2016). Translators at War. K International. [En línea: https://www.kinternational.com/blog/translators-at-war/. Fecha de consulta: 12/02/2019].

Calduch, R. (1993). Dinámica de la sociedad internacional. Madrid: Centro de Estudios Ramón Areces.

Casado, M. (1991). Lenguaje y Cultura. Madrid: Síntesis.

Diccionario de la Real Academia Española (22 $2^{\mathrm{a}}$. Ed). Disponible en Web: $<$ http://www.rae.es>

Eco, U. (1986). Golpear el corazón del Estado. La estrategia de la ilusión. Barcelona: Lúmen.

Fundéu [en línea]. Disponible en Web: < https://www.fundeu.es/ >.

Gadarian, S. (2010). The politics of threat: How terrorism news shapes foreign policy attitudes. The Journal of Politics 72 (2), pp. 469-483.

Hoffman, Bruce. A mano armada. Historia del terrorismo. Madrid: Espasa-Calpe, 1990.

Kenny, D. (200I). Lexis and Creativity in Translation. A corpus-based study Manchester: St. Jerome.

Laviosa, S. (1998). The English Comparable Corpus: a Resource and a Methodology. En Unity in Diversity: Current Trends in Translation Studies. Manchester: St. Jerome Publishing.

Robles, F. (2007). El problema de los traductores en la guerra global contra el terrorismo. Puentes 8, pp. 33-40.

Rodrigo, M. (1991). Los medios de comunicación ante el terrorismo. Barcelona: Icaria.

Shariffan, F. (2007). Politics and/of Translation: Case Studies between Persian and English. Journal of Intercultural Studies 28 (4), pp. 4I 3-424.

Torres, H. (20l0). El terrorismo, su inexistencia o inoperancia: la apertura a la violación de los derechos humanos. Diálogos de Saberes, pp. 77-90.

Veres, L. (2006). La retórica del terror. Sobre lenguaje, terrorismo y medios de comunicación. Madrid: Logos.

Veres, L. (20I2). Imagen, terrorismo y argumentación. Revista Iberoamericana de Argumentación, 4, pp. I-I4. 
